\title{
GATTACA: Lightweight Metagenomic Binning with Compact Indexing of Kmer Counts and MinHash-based Panel Selection
}

\author{
Victoria Popic $^{1 \star}$, Volodymyr Kuleshov ${ }^{1}$, Michael Snyder ${ }^{2}$, and Serafim Batzoglou ${ }^{1 *}$ \\ 1 Department of Computer Science, Stanford University, Stanford CA, USA \\ ${ }^{2}$ Department of Genetics, Stanford University, Stanford CA, USA \\ \{viq, kuleshov, mpsnyder, serafim\}@stanford.edu
}

\begin{abstract}
We introduce GATTACA, a framework for rapid and accurate binning of metagenomic contigs from a single or multiple metagenomic samples into clusters associated with individual species. The clusters are computed using co-abundance profiles within a set of reference metagnomes; unlike previous methods, GATTACA estimates these profiles from k-mer counts stored in a highly compact index. On multiple synthetic and real benchmark datasets, GATTACA produces clusters that correspond to distinct bacterial species with an accuracy that matches earlier methods, while being up to $20 \times$ faster when the reference panel index can be computed offline and $6 \times$ faster for online co-abundance estimation. Leveraging the MinHash technique to quickly compare metagenomic samples, GATTACA also provides an efficient way to identify publicly-available metagenomic data that can be incorporated into the set of reference metagenomes to further improve binning accuracy. Thus, enabling easy indexing and reuse of publicly-available metagenomic datasets, GATTACA makes accurate metagenomic analyses accessible to a much wider range of researchers.
\end{abstract}

\section{Introduction}

Despite their important role, microbes constitute the dark matter of the biological universe. Thousands of species live in the human gut, but only a small fraction can be isolated and studied in a laboratory and very little is known about those that cannot be cultured. The short read lengths of modern sequencing instruments - combined with various inherent difficulties associated with complex bacterial environments - make it very difficult to perform simple tasks such as accurately identifying bacterial strains, recovering their genomic sequences, and assessing their abundance. Many approaches have been proposed to address these shortcomings. Specialized library preparation techniques such as Hi-C or synthetic long reads are often very accurate, but also prohibitively complex. As a result, approaches based on contig binning are more popular in practice. Metagenomic binning refers to the problem of grouping together partially assembled sequence fragments (or contigs) that belong to the same species. Current binning techniques fall into mainly two categories: (1) supervised classification of contigs into known taxons via comparisons to previously catalogued species $13,26,30$ and (2) unsupervised clustering techniques using features derived directly from the metagenomic sample data [?, 2, 3, 16, 17, 20, 31, where unsupervised clustering has the clear advantage of binning contigs that pertain to previously unknown species. While some unsupervised techniques 17, 28 perform clustering based only on the contig sequence composition (the frequency of certain short motifs, e.g. all tetra-mers), the most successful recent approaches [2, 3, 16, 20, 31 also incorporate contig coverage profiles across multiple metagenomic samples. In brief, these techniques assemble de-novo bacterial contigs and estimate the coverage of each contig within each sample of a large mategenomic cohort using read mapping. Naturally, contigs belonging to the same species will have similar abundances across different samples (determined by which cohort samples the species is present in); coverage profiles can therefore

\footnotetext{
* Corresponding author.
} 
be used to cluster related contigs. This approach is accurate but has two main limitations: it requires a large cohort of samples, as well as sizable compute resources for read alignment. We address both of these limitations in this work.

In particular, we present GATTACA, a lightweight framework for metagenomic binning, which (1) avoids read alignment without loss of accuracy and (2) enables efficient stand-alone analysis of single metagenomic samples. Both results are based on the finding that we can approximate contig coverages using kmer counts while still achieving the same binning accuracy as leading alignment-based methods. In addition to offering a significant speedup in coverage estimation, using kmer counts, as opposed to alignment, provides us with the exciting ability to index offline any publicly-available metagenomic sample and incorporate it into the coverage profile of the contigs being processed. This allows us to efficiently pull in data from large growing repositories, such as the Human Microbiome Project (HMP) 29] or EBI Metagenomics archive [12 into any metagenomic study (especially one where only a single or few samples are available) at almost no cost. For example, our kmer count index for a typical HMP sample only requires 100MB on average. We achieve the small space requirement by leveraging memory-efficient hashing with minimal perfect hash functions (MPHFs) and the probabilistic Bloom filter data structure. In contrast, using these datasets with read alignment would require massive downloads (for example, a single HMP sample is roughly 7GB compressed and 30GB uncompressed) and expensive subsequent handling to map the reads. In terms of speedup, we found our coverage estimation time to be at least an order of magnitude faster (approximately $20 \times$ ) when the index is computed offline (e.g. for recyclable public reference samples) and about about $6 \times$ when the kmers are counted on-the-fly (e.g. for private samples used only once), when compared to read mapping.

While using small indices allows us to incorporate a large number of publicly-available samples into a given study, not all existing samples will carry content relevant to the study in question. Namely, samples that don't contain any of the species present in a given set of contigs cannot contribute any useful information for grouping the contigs. The same logic applies also to samples that carry content identical to a sample that has already been included. This motivates the need to additionaly define appropriate sample selection criteria, for which we propose two metrics: (1) relevance and (2) diversity. More specifically, we would like to select a panel of samples which share content with the sample being analyzed (our query) but that also differ in the content that is shared. We use locality sensitive hashing [15] and the MinHash technique 7], to compare the samples efficiently. At a high level, we create and index small MinHash fingerprints for each sample in the database (offline), and then extract the appropriate samples according to the fingerprint of the query. The resulting index can be separately downloaded and used to determine which samples to include into the analysis; it needs to be updated only occasionally when new samples become available.

We evaluate GATTACA in clustering contigs assembled across multiple samples (co-assemblies) and from individual samples, using both synthetic and real datasets. We compare our results with several state-of-the-art methods in metagenomic binning: CONCOCT [3], MetaBat [16, and MaxBin [31], using standardized cluster evaluation metrics and benchmarks (reusing evaluation scripts from existing methods when appropriate). GATTACA was implemented in $\mathrm{C}++$ and Python and is freely available at http: : //viq854.github.com/gattaca.

\section{Methods}

\subsection{Index of Kmer Counts}

In order to quickly estimate contig coverages, GATTACA builds a small index of kmer counts for each sample in the cohort. Several solutions have been proposed for exact (e.g. using hash maps [21] or minimum perfect hash functions [24]) and approximate kmer counting (e.g. using the count-min sketch [32]). Since the content of each sample in our panel is static, our index uses a minimal perfect hash function 9 ] to store the kmer counts without loss of accuracy, resulting in a drastic reduction in space when compared to traditional 
hash tables (we also found it to be more space-efficient than the count-min sketch solution for the same binning accuracy). At a high level, given a set $S$ of $n$ keys, a minimal perfect hash function (MPHF) $h$ provides a mapping between the keys and $n$ consecutive integers from 0 to $n-1$; that is, $h$ is an injection on $S$, guaranteeing no collisions among its keys (for $x$ and $y$ in $S$, if $x \neq y$, then $h(x) \neq h(y)$ ) and exactly $n$ possible outputs from the integer set $\{0,1,2, \ldots, n-1\}$. We use the BDZ algorithm based on random $r$-partite hypergraphs [6] for constructing the MPHFs.

Index Construction. To construct the index, we first generate the kmers from the all reads in the sample (accounting for both forward and reverse complement strands) and exclude kmers that occur only once, since these are most likely present due to sequencing errors. We use a kmer length of 31-bp in our experiments (compacting the kmers into 64-bit integers for convenience). We then generate the MPHF, $h_{S}$, for the resulting set of distinct kmers, $S$, and store their counts in an integer array $A(|A|=|S|)$, at the indices given by $h_{S}$; namely, $A\left[h_{S}(x)\right]=\operatorname{count}(x)$, for each kmer $x \in S$. We found 8 bits to be sufficient for storing the kmer counts (and since many counts are small, these can be compressed even further using techniques such as varint encoding). Finally, we need to store the elements in $S$ to support lookups, since $h_{S}(z)$ for $z \notin S$ will return a valid but incorrect index into $A$. One direct solution for storing $S$ would be to rely on the MPHF, using a secondary array $B$ and setting $B\left[h_{S}(x)\right]=x$ for all kmers $x \in S$; then we could check upon lookup of a key $y$, if $B\left[h_{S}(y)\right]$ is equal $y$ and determine whether $y$ was in the set. However, this solution requires storing the array $B$ of $|S| 64$-bit integers, which is $4 \times$ larger than $A$, and would substantially increase the index. So instead, we store the set $S$ in a Bloom filter, $B F$, which is a widely used probabilistic data structure for testing set membership that offers space-efficiency at the expense of possible false positives (no false negatives are possible). We have configured the size of $B F$ based on a false positive probability of 0.05. As a result, our index for each sample consists of: (1) the MPHF, $h_{S},(2)$ the array of counts, $A$, and (3) the Bloom filter storing the elements of $S, B F$. As an example, the size of the index constructed for an HMP sample containing 20 million 100 -bp long reads was 108MB.

Coverage Estimation. Given a contig $c$ and an index $I$ of a cohort sample, we estimate the coverage of $c$ in this sample by performing lookups in $I$ for each kmer in $c$ and then computing the median of the resulting counts. More specifically, we return the median of the set of counts $C=\{\ldots \operatorname{count}(x) \ldots . \mid \forall \operatorname{kmers} x \in C\}$, where

$$
\operatorname{count}(x)= \begin{cases}I \cdot A\left[I \cdot h_{S}(x)\right], & \text { if } x \in I \cdot B F \\ 0, & \text { otherwise }\end{cases}
$$

\subsection{Contig Representation}

Given a set of contigs assembled from a single or multiple metagenomic samples, our goal is to bin together the contigs that belong to the same class (e.g. species or strain). Similar to existing methods, e.g. CONCOCT, we first represent each contig as a multi-dimensional vector using both its sequence composition and coverage profile across multiple samples, where our coverages are approximated using kmer counts instead of read mapping, as described above. Namely, given $M$ reference samples (either from the same study or from a public database), our coverage profile is the median count of the contig kmers in each sample., while the composition profile is the normalized frequency of each possible tetra-mer in the contig and its reverse complement (resulting in a total of $F=136$ such features); the normalization of composition features is done according to the CONCOCT procedure (please see 3 for details). Therefore, each contig is a vector $V=\left[c_{1}, \ldots, c_{M}, f_{1}, \ldots, f_{F}\right]$, where $c_{i}:=$ the median kmer count in sample $i$ and $f_{j}:=$ the frequency of tetra-mer $j$ in the contig sequence. 


\subsection{Clustering algorithm}

Given the resulting vector representations, we cluster the contigs using a Bayesian Gaussian mixture model (GMM) with a Dirichlet prior. In brief, we define a mixture distribution $p$ of $K$ Gaussian components over $n$ data points $x_{i} \in \mathbb{R}^{d}$ and (unobserved) assignment labels $z_{i} \in\{0,1\}^{K}$ for $i=1, \ldots, n$. Our model is the product of a likelihood term

$$
p(z, X \mid \theta)=\prod_{i=1}^{n} \prod_{k=1}^{K} \pi_{k}^{z_{i k}} \mathcal{N}\left(x_{i} \mid \mu_{k}, \Lambda_{k}^{-1}\right)^{z_{i k}}
$$

and a prior term

$$
p(\theta)=\operatorname{Dir}\left(\pi \mid \alpha_{0}\right) \prod_{k=1}^{K} \mathcal{N}\left(\mu_{k} \mid m_{0},\left(\beta_{0} \Lambda_{k}\right)^{-1}\right) \mathrm{Wi}\left(\Lambda_{k} \mid L_{0} \nu_{0}\right) .
$$

Here, $X \in \mathbb{R}^{d \times n}$ is the matrix of data points and $\mathcal{N}\left(\cdot \mid \mu_{k}, \Lambda_{k}^{-1}\right)^{z_{i k}}$ is a multivariate Gaussian with mean $\mu_{k}$ and inverse covariance matrix $\Lambda_{k}$. The $\pi \in \Delta_{K-1}$ form a vector of cluster weights. Together, the $\mu_{k}, \Lambda_{k}, \pi$ form the parameter vector $\theta$ of the likelihood. The prior over $\theta$ is a product of a Dirichlet with hyperparemeter $\alpha_{0} \in \Delta_{K-1}$, a multivariate normal with hyper-parameters $m_{0} \in \mathbb{R}^{d}, \beta_{0}>0$ and a Wishart distribution parametrized by $L_{0} \in \mathbb{R}^{d \times d}$ p.s.d. and $\nu_{0}>0$.

We perform inference by maximizing the marginal $\log$-likelihood $\log p(X)$ using variational inference. In brief, we maximize the evidence lower bound

$$
\log p(X) \geq \mathbb{E}_{q(z, \theta)}[\log p(z, X, \theta)-\log q(X, z)]
$$

over the set of approximating distributions $q$. By our choice of conjugate prior, the posterior $p(\theta, z \mid X)$ and hence the optimal $q$ have the same form, which factors over $q(z \mid \theta) q(\theta)$. We optimize the bound using variational expectation-maximization, which consists of repeatedly choosing updating $q(z \mid \theta)$ and $q(\theta)$. Each update has a closed-form solution by our choice of conjugate prior. We conclude the algorithm by assigning each data point to its maximum a-posteriori label according to $q(z \mid \theta)$. We refer the reader to section 21.6.1 in the standard textbook of Murphy (2012) 22 for the full derivation of this algorithm.

At a high-level, the above model is very similar to automatic relevance determination (ARD), which is used by CONCOCT. We have found our approach to perform better in practice than ARD, especially for automatically determining the number of clusters in the data. Both algorithms are implemented in our software package. Other alternative clustering methods can also be easily plugged into GATTACA's binning pipeline.

\subsection{Sample Selection}

Given a query sample, $Q$, we would like to select $n$ samples from the public database, which can provide discriminatory features for clustering the contigs of $Q$ (where the features represent the coverage of the contigs in the respective samples). Intuitively, the selected samples must share some content with $Q$ (have relevance), as well as have pairwise diversity among themselves to guarantee coverage of different contigs of $Q$. Similar relevance and diversity concepts can be found in online recommendation systems (e.g. for articles or music [1,8]).

By representing each sample as a set of overlapping kmers, we apply the Jaccard coefficient to measure their similarity, where the Jaccard coefficient $J(A, B)=\frac{|A \cap B|}{|A \cup B|}$, for two sets $A$ and $B$. Then, we can consider relevant the samples that are within a certain distance from $Q$ under Jaccard (e.g. all samples $S$ for which $J(S, Q)>0)$. However, computing the Jaccard distances directly on the kmer sets would be inefficient for large databases. Therefore, we apply the min-wise independent permutations (MinHash) LSH scheme [7] to create small fingerprints for each sample set instead, defined as follows. 
Let $U$ be the ground set of all possible set items. Given a random permutation $\pi$ of indices of $U$ and a set $X$, let $h_{\pi}(X)=\min _{x \in X}\{\pi(x)\}$. The MinHash LSH family $H$ will consist of all such functions for each choice of $\pi$. It can be easily shown that for a given $h$ chosen uniformly at random, $\operatorname{Pr}\left[h_{\pi}(A)=h_{\pi}(B)\right]=J(A, B)$ (see 7 for details). Due to the high variance in the probability of collision, we concatenate $L$ different hash functions from the family $H$ chosen independently at random to form the fingerprint. Then given the number of the hash collisions among the chosen $L$ functions, $c$, the ratio $c / L$ can also be used as an unbiased estimator for $J(A, B)$.

To summarize, given the kmer set $K=\left\{s_{0}, s_{1}, \ldots, s_{n-1}\right\}$ of some sample $S$ and $L$ hash functions from $H$, we construct the MinHash fingerprint vector $F=\left[f_{0}, f_{1}, \ldots, f_{L-1}\right]$, such that the fingerprint entry $f_{i}$ is the minimum set element under hash function $h_{i}$ :

$$
f_{i}=\min \left\{h_{i}\left(s_{0}\right), h_{i}\left(s_{1}\right), \ldots, h_{i}\left(s_{n-1}\right)\right\} .
$$

Now given the fingerprints, we can define relevance between a sample $S$ and $Q$ as simply the number of entries shared by their fingerprints. By indexing the fingerprints of all the samples in the database into $L$ tables (based on the value of each fingerprint entry, respectively), we can find all the samples that share at least one fingerprint entry in common with $Q$ using simple lookups, as well as rank them according to relevance.

Finally, if the number of relevant samples is too high, we can reduce our panel using the diversity criterion. That is, given all the relevant samples, we can select $n$ samples that maximize the diversity of the set. This problem is known as the dispersion problem [10, where the objective is to locate $k$ points among $n$, such that some function of distances between the $k$ points is maximized. One popular optimality criteria is the MAX-MIN, which maximizes the minimum distance between a pair of points. This problem is known to be NP-hard; however, an efficient greedy heuristic algorithm exists for the MAX-MIN dispersion problem when the distances satisfy the triangle inequality, with provable performance guarantee of 2 25]. Given two samples $A$ and $B$, we define their diversity as: $D(A, B)=1-J(A, B)$ and apply the greedy algorithm of $[25$ to find the $n$ samples. While this procedure is simple and can be efficiently used to detect samples with distinct kmer sets, its main limitation is that it cannot be used to find samples which differ in kmer frequency only (since frequency does not affect Jaccard distance), which could also be used to generate discriminatory features.

\section{Results}

\subsection{Datasets}

Synthetic datasets. We used two synthetic datasets generated by Alneberg et al. 3 from the 16S rRNA samples of the Human Microbiome Project(HMP) [29. The first dataset ("Species-Mock") consists of 96 samples containing a mixture of 101 different species (without strain-level variation), while the second dataset ("Strain-Mock") consists of 64 samples comprising a mixture of 20 different organisms, of which some represent strains of the same species (e.g., this dataset includes five different E. coli strains). The relative abundance profiles of the species and strains in each sample were assigned according to the distribution of the 101 and 20 most abundant organisms in the original HMP samples, respectively. Reads (100-bp long) were simulated from random positions of the genomes present in the sample based on their relative abundance, for a total of 7.75 million reads and 11.75 million reads in each "Species-Mock" and "Strain-Mock" sample, respectively. Both datasets contain the set of contigs co-assembled across all the samples by Alneberg et al. using the Ray assembler [5], and partitioned into fragments of 10 kilobases when appropriate. We used the default minimum contig length of 1000-bp when running CONCOCT, MaxBin, and GATTACA; this parameter was set to $1500-\mathrm{bp}$ for MetaBat, which is the smallest length supported by this method. As a result, the "Species-Mock" included 37,627 valid contigs and the "Strain-Mock" included 9,411 valid contigs 
(for all tools except MetaBat). To determine the genome assignment for each contig, the simulated reads were mapped back to the contigs and the genome was selected based on the majority of the mapped reads (with the requirement that it corresponds to at least $50 \%$ of the reads and there are at least 10 unambiguously mapped reads); this is the same criteria using by Alneberg et al..

To evaluate single-sample binning, we also assembled the contigs for each simulated sample of the "Species-Mock" independently. We used the SPADES [4] assembler with default parameters and automatic $\mathrm{k}$-mer selection, and applied the same post-processing and labeling criteria.

Real datasets. To evaluate co-assembly based binning we used the small Sharon et al. (2013) dataset comprised of 11 fecal samples from a preterm infant 27 . We downloaded $1.372 \times 10^{8} 100$-bp short reads from the SRA052203 NCBI archive as 18 separate samples (of which 7 were resequenced from the original 11 samples). The co-assembled contigs for this dataset were made public by Alneberg et al. 3]. To test binning accuracy, we used the CheckM 23] method based on single-copy core genes (SCGs), which are housekeeping genes that occur in single copies in the microbial genomes and have been previously used to evaluate the purity of the metagenomic clusters 3 .

To evaluate binning of contigs assembled from single samples, we downloaded Illumina raw sequencing reads from the following two publicly-available gut microbiome sample collections: (1) all the available gut samples from HMP (148 total) and (2) 56 gut samples from the Nielsen et al. study 2 available through the European Nucleotide Archive. We used the SPADES 4 assembler with default parameters to assemble the contigs of individual samples (cutting contigs into 10 kilobase fragments and filtering contigs shorter than 1000-bp, as in simulation). Since the HMP samples were very large (over 30GB/sample uncompressed), we subsampled them using the seqtk program 11] to 20 million paired-end reads per sample during index construction. To label the contigs in each evaluated sample we used the TAXAssign program. Labels were assigned based on the top 100 hits against the BLAST NCBI genome database that had at least 95\% identity and $90 \%$ query coverage. A taxonomic label was assigned if $90 \%$ of the hits at a given level belong to the taxon associated with that label. This procedure classified roughly $15 \%$ of them on average at the species level depending on the sample.

Since only a small fraction of contigs could be classified via TAXAssign, we also used SCGs to evaluate binning accuracy. In addition to CheckM, we also applied Prodigal 14 to predict and functionally annotate genes on our sample contigs and then RPS-BLAST to COG annotate the protein sequences (using the NCBI COG database). We then used the 36 Clusters of Orthologous Groups (COGs) pre-selected by Alneberg et al., along with their script COG_table.py to generate the number of SCGs in each contig cluster.

\subsection{Clustering Evaluation Metrics}

We apply the following three standardized metrics for evaluating metagenomic clustering of labelled contigs (e.g simulated contigs for which we know the ground-truth genome assignment or contigs confidently classified using TAXAssign): (1) recall - measures how many same-class contigs are placed in the same cluster, (2) precision - measures the purity of each cluster, and (3) adjusted Rand index (ARI) - measures how often pairs of same-class contigs are clustered together. Let $N$ be the total number of contigs, $K$ be the number of computed clusters, and $S$ be the number of contig labels in the data (e.g. different species or strains). Also let $n_{k s}$ be the number of contigs in the $k$-th cluster with label $s$. Then we have:

1. recall $=\frac{1}{N} * \sum_{s} \max _{k}\left\{n_{k s}\right\}$

2. precision $=\frac{1}{N} * \sum_{k} \max _{s}\left\{n_{k s}\right\}$

3. ARI $=\frac{\sum_{s, k}\left(\begin{array}{c}n_{k s} s \\ 2\end{array}\right)-\frac{\sum_{k}\left(\begin{array}{c}n_{k} \\ 2\end{array}\right) \sum_{s}\left(\begin{array}{c}n_{i} s \\ 2\end{array}\right)}{\left(\begin{array}{c}N \\ 2\end{array}\right)}}{\frac{1}{2}\left[\sum_{k}\left(\begin{array}{c}n_{k} \\ 2\end{array}\right)+\sum_{s}\left(\begin{array}{c}n_{2} s \\ \dot{2}\end{array}\right)\right]-\frac{\sum_{k}\left(\begin{array}{c}n_{k} \\ 2\end{array}\right) \sum_{s}\left(\begin{array}{c}n_{2} \\ 2\end{array}\right)}{\left(\begin{array}{c}N \\ 2\end{array}\right)}}$, where $n_{. s}=\sum_{k} n_{k s}$ and $n_{k}=\sum_{s} n_{k s}$. 


\subsection{Synthetic Benchmarks}

First we show that replacing read mapping with kmer counting for coverage estimation does not result in loss of clustering accuracy using two synthetic benchmarks created by Alneberg et al. 3. Namely, we compare the performance of GATTACA, CONCOCT, MaxBin, and MetaBat on the "Species-Mock" and "Strain-Mock" datasets, using the published co-assembled contigs. Here we build the index of kmer counts only from the cohort of samples associated with each dataset. As a result, our "Species-Mock" index contains 96 samples (with a total size of 12GB) and our "Strain-Mock" index contains 64 samples (size of $5.1 \mathrm{~GB}$ ). Table 1 shows the accuracy results for each tool (computed with the Validate.pl script provided by CONCOCT). GATTACA achieves similar recall to CONCOCT and MaxBin (all higher than MetaBat) on both datasets, while its precision and ARI are somewhat lower on the "Species-Mock" and higher on the "Strain-Mock". MetaBat achieves the highest precision on both datasets; however, it clusters substantially fewer contigs.

\begin{tabular}{|l|c|c|c|c|c|c|c|c|}
\hline Dataset & Contigs & Labeled contigs & True clust. & Pred. clust. & Rec & Prec & Adj. Rand Idx. & Program \\
\hline Species-Mock & 37,628 & 37,589 & 101 & 102 & 0.993 & 0.978 & 0.971 & GATTACA \\
& & & & 101 & 0.998 & 0.988 & 0.983 & CONCOCT \\
& & & & 101 & 0.997 & 0.989 & 0.982 & MaxBin \\
& 37,501 & 37,490 & 101 & 139 & 0.950 & 0.999 & 0.953 & MetaBat \\
\hline Strain-Mock & 9,417 & 9,109 & 20 & 22 & 0.975 & 0.968 & 0.973 & GATTACA \\
& & & & 21 & 0.982 & 0.942 & 0.945 & CONCOCT \\
& & & & 116 & 0.967 & 0.917 & 0.879 & MaxBin \\
& \multirow{2}{*}{6,281} & 6,244 & 20 & 34 & 0.856 & 0.998 & 0.857 & MetaBat \\
\hline
\end{tabular}

Table 1: Binning performance on synthetic data. Synthetic datasets were obtained by generating reads from known genomes; bacteria differ either at the strain or at the species level.

Since one of the goals of this project is to support single-sample analysis, we also evaluate single-sample contig clustering on simulated data. In particular, we assembled several samples of the synthetic "SpeciesMock" dataset separately and used the remaining samples to build the index of kmer counts in a "leave-outout" fashion; namely, for a given sample $Q$ our index includes all the samples in the original cohort except $Q$. Table 2 shows the performance on three different samples, demonstrating the accuracy of our approach.

\begin{tabular}{|c|c|c|c|c|c|c|c|}
\hline Sample & Ctgs & Labeled Ctgs. & True clust. & Pred. clust. & Rec & Prec & Adj. Rand Idx. \\
\hline S1023 & 8,245 & 8,237 & 25 & 19 & 0.964 & 0.985 & 0.952 \\
\hline S1063 & 12,845 & 12,824 & 25 & 25 & 0.991 & 0.932 & 0.919 \\
\hline S1095 & 12,863 & 12,848 & 31 & 28 & 0.996 & 0.995 & 0.996 \\
\hline
\end{tabular}

Table 2: Leave-one-out accuracy of GATTACA on the "Species-Mock" dataset. We assemble contigs from a single sample and cluster them with a reference index constructed from all the other samples. Performance is comparable to when all contigs were co-assembled.

\subsection{Real Data Benchmarks}

Next we evaluate binning on the real Sharon co-assembly. Since the true reference genomes are not available for this experiment, we use the CheckM [23] method based on SCGs to approximate recall and precision. The Sharon dataset consists of 18 samples and the resulting GATTACA index has a size of $2.2 \mathrm{~GB}$. Figure 1 shows the number of genomes identified by each method with a precision higher that $90 \%$ and $95 \%$, 
bioRxiv preprint doi: https://doi.org/10.1101/130997; this version posted April 26, 2017. The copyright holder for this preprint (which was not certified by peer review) is the author/funder, who has granted bioRxiv a license to display the preprint in perpetuity. It is made available under aCC-BY-NC 4.0 International license.

respectively, and varying recall, as reported by CheckM. All methods, except MetaBat, produce 3 highlyaccurate genomes with precision and recall $\geq 95 \%$ (MetaBat generates only one such genome). GATTACA's performance is very similar to CONCOCT here, with the exception of an unreported bin at precision $\geq 90 \%$, which CONCOCT produces with a recall of $30 \%$.

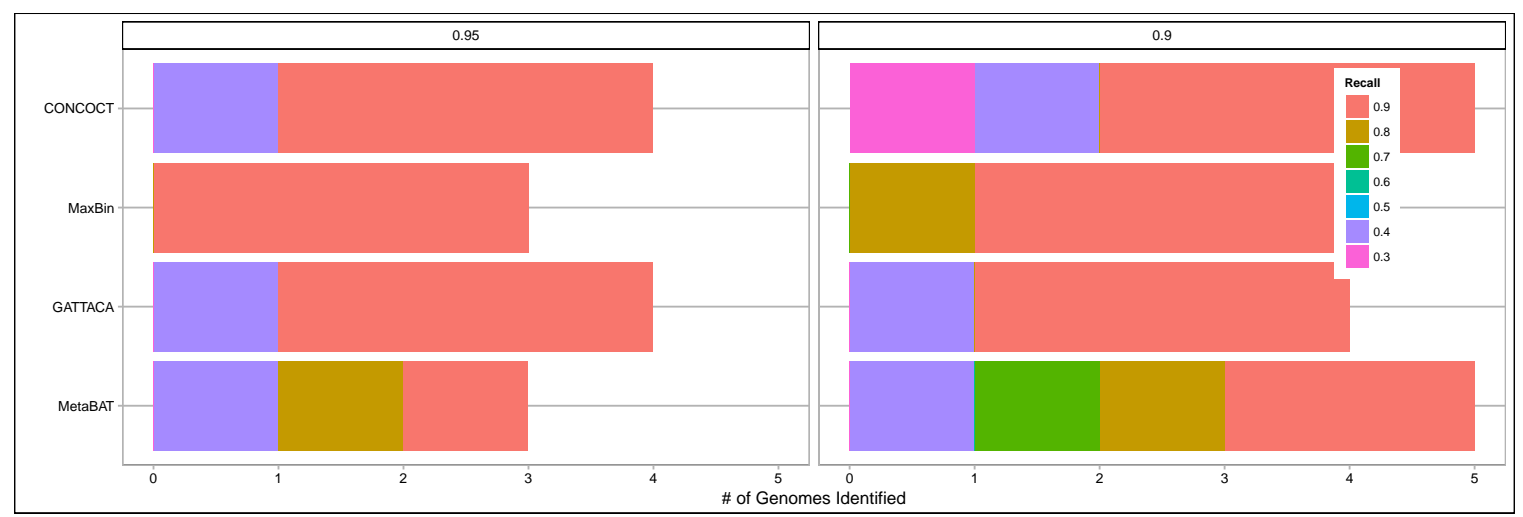

Fig. 1: Binning performance on the real Sharon dataset reported by CheckM 23. The number of genomes identified with $\geq 90 \%$ (left) and $\geq 95 \%$ (right) is shown for varying recall. The image was generated the 'benchmark. R' script (slightly modified) provided by MetaBat.

We also evaluate binning of single-sample assemblies using a panel of publicly available datasets of the gut microbiome. Table 3 shows the binning accuracy computed using TAXAssign labels for three HMP stool samples clustered using a panel of 95 different stool samples downloaded from the HMP database. Due to the long read mapping runtime, we show results for CONCOCT, MetaBat, and MaxBin on only one of the HMP samples, SRS011239. Figure 2 also shows the SCG-based results for SRS011239 obtained using CheckM.

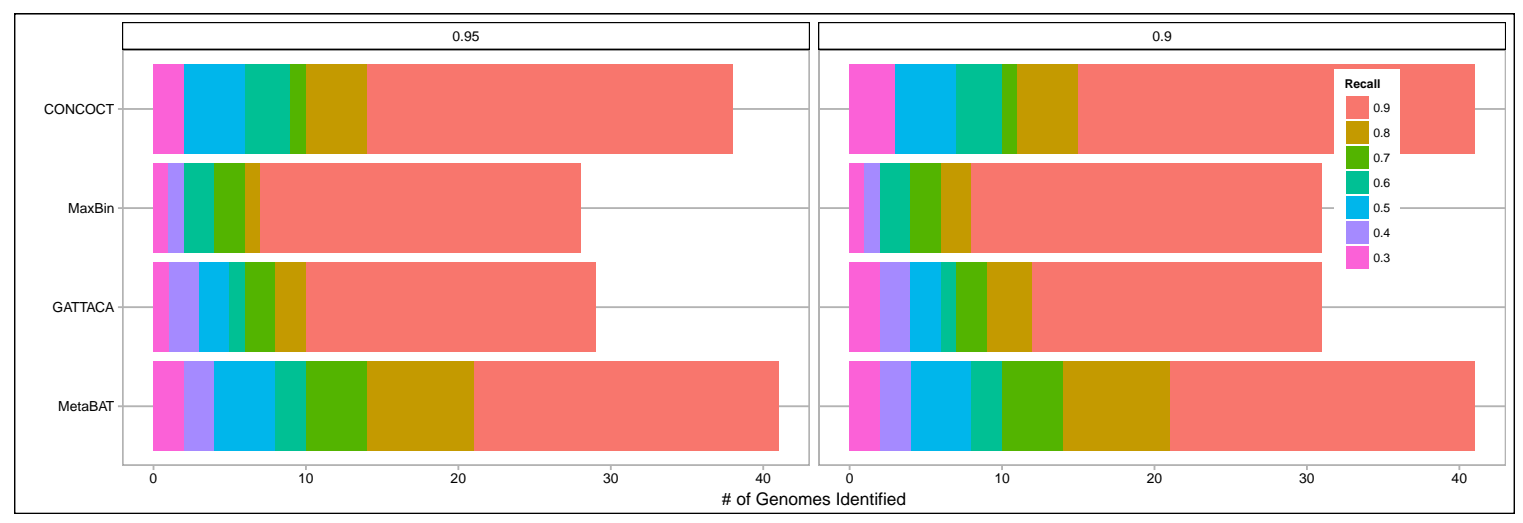

Fig. 2: Binning performance on the real SRS011239 sample and a panel of 95 HMP metagenomes reported by CheckM 23]. The number of genomes identified with $\geq 90 \%$ (left) and $\geq 95 \%$ (right) is shown for varying recall. The image was generated the 'benchmark.R' script (slightly modified) provided by MetaBat. 


\begin{tabular}{|l|c|c|c|c|c|c|c|c|}
\hline Dataset & Ctgs & Labeled Ctgs & True Clust. & Pred. Clust. & Rec & Prec & Adj. Rand idx. & Program \\
\hline SRS011239 & 28,839 & 5,430 & 46 & 131 & 0.910 & 0.963 & 0.929 & GATTACA \\
& 33,180 & 5,456 & 48 & 212 & 0.902 & 0.959 & 0.906 & CONCOCT \\
& 33,166 & 5,456 & 48 & 52 & 0.928 & 0.936 & 0.910 & MaxBin \\
& 16,136 & 3,419 & 17 & 51 & 0.920 & 0.988 & 0.937 & MetaBat \\
\hline SRS011134 & 37,645 & 5,200 & 54 & 128 & 0.848 & 0.920 & 0.892 & GATTACA \\
\hline SRS011302 & 28,034 & 4,712 & 55 & 115 & 0.875 & 0.929 & 0.898 & GATTACA \\
\hline
\end{tabular}

Table 3: Clustering of real HMP samples using a panel of 95 other HMP metagenomes. Performance is stable and accurate across samples, with an adj. Rand index of about 90\%. True assignments were obtained using TAXAassign. Contigs that were not covered by at least a single sample were not included in the clustering step.

Next we evaluate GATTACA in clustering contigs from a single sample of the Nielsen et al. 2] study using an index built from 46 samples of the study and augmented with 83 HMP samples selected using the MinHash sample index based on the relevance criteria described above. Table 4 show the results based on the TAXAssign labels. We also show the results when using the 46 samples of the study only. It can be seen that the binning precision improves significantly with the addition of the HMP samples, motivating the inclusion of publicly-available data in studies with fewer available samples.

\begin{tabular}{|l|c|c|c|c|c|c|c|}
\hline Index & Ctgs. & Labeled Ctgs. & True Clust. & Pred. Clust. & Rec & Prec & Adj. Rand idx. \\
\hline Nielsen & 30,345 & 2,655 & 45 & 97 & 0.699 & 0.833 & 0.660 \\
\hline Nielsen+HMP(83) & 30,821 & 2,658 & 45 & 129 & 0.711 & 0.882 & 0.735 \\
\hline
\end{tabular}

Table 4: Binning sample SAMEA1965191 from the study of Nielsen et al. 2 2] using an index of samples from the study and an index augmented with HMP samples. Increasing the reference size greatly improves clustering accuracy. Contigs that were not covered by at least a single sample were included in the clustering step.

\subsection{Evaluation of Sample Selection with MinHash}

In order to efficiently leverage publicly-available data, we apply the MinHash technique to quickly identify public samples that are relevant (i.e. share content) to a given metagenomic study. In particular, we quickly compare the fingerprint(s) of the study's single or multiple samples, to the fingerprints (indexed for efficiency) of publicly-available metagenomic datasets. We found that MInHash fingerprints of length 1024 provide a good tradeoff between runtime and sensitivity. This requires only $8 \mathrm{~K}$ additional space per metagenomic sample to store its fingerprint. In particular, we performed the following experiment to evaluate the performance of MinHash for this task. Given a randomly-selected real query sample $Q$ (namely, SRS011134 from the HMP), we created a panel of 50 samples (each containing 10M reads of length 100-bp) that share a varying fraction of reads with $Q$, ranging from $2 \%$ to $100 \%$. More specifically, we first started with $Q$ and 49 samples containing reads simulated from the chicken genome (under the assumption that there will be little content similarity between this genome and the species present in SRS011134). We then replaced varying fractions of the chicken reads with the reads of SRS011134. Figure 3 shows the true Jaccard similarity between the panel samples and $Q$, as well as the Jaccard similarity estimated using MinHash, with varying fingerprint lengths.

To further analyze the power and efficiency of using MinHash for metagenomic sample comparison, we downloaded $32 \mathrm{HMP}$ samples originating from different body sites (e.g. gut, saliva, throat). We then computed the MinHash fingerprints (of length 1024) of each sample and the Jaccard distances estimated 


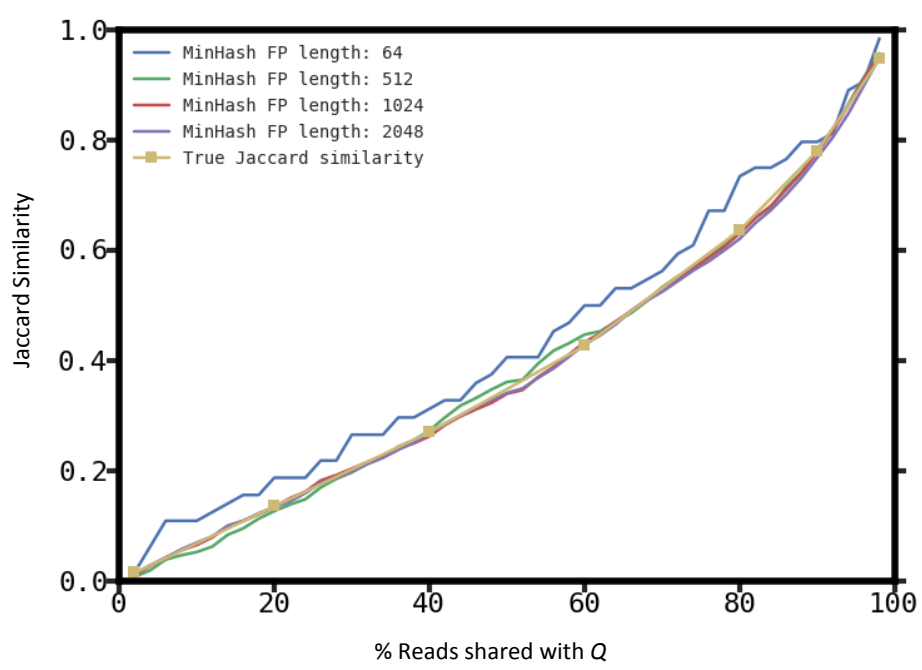

Fig. 3: Jaccard similarities estimated using MinHash for a query sample $Q$ (SRS011134) and a panel of 49 samples sharing varying levels of content similarity with $Q$. The true Jaccard similarity is shown along with estimates computed from fingerprints of different length.

using these fingerprints. Figure 4 shows the resulting distance matrix and hierarchical clustering of these 32 samples. As expected, samples originating from the same body site are significantly more similar in content, as reflected by the MinHash fingerprints, and cluster together. The pairwise distance computations performed in this experiment took less than a second total, and can easily scale to large datasets of publicly available metagenomic datasets; suggesting that MinHash is a good candidate for the quick and accurate metagenomic sample comparison.

\subsection{Runtime Performance}

Finally we compare the runtime of the CONCOCT pipeline based on read mapping (which internally uses Bowtie2 [18, samtools [19, and Picard tools) to the GATTACA kmer counting implementation for estimating the coverage of pre-assembled contigs of a single HMP sample (SRS011134). The CONCOCT runtime is split as follows: $10.1 \mathrm{~min}$ for BWT index construction (from the contigs) and $51.2 \mathrm{~min}$ for coverage estimation in one sample. The GATTACA runtime consists of $5.3 \mathrm{~min}$ in kmer count index construction and $2.7 \mathrm{~min}$ for lookups in one sample. Therefore, ignoring the BWT indexing time, it would take CONCOCT roughly $81 \mathrm{~h}$ to map the 95 HMP samples against these contigs; while GATTACA would require only $12.7 \mathrm{~h}$. However, if the samples in the cohort have already been indexed (for publicly available data or multi-sample studies reusing the same sample cohort), then GATTACA would only need $4.3 \mathrm{~h}$ to finish (resulting in a roughly $20 \times$ speedup). The reported times were obtained running on a single $2.67 \mathrm{GHz}$ Intel Xeon X5550 processor (in single-threaded mode). Both of the tools can be easily run in parallel across multiple samples, contigs, or sample reads.

\section{Conclusion}

GATTACA is a lightweight method for metagenomic binning, which achieves comparable binning accuracy with leading alignment-based methods at a fraction of the cost by approximating contig abundances using 


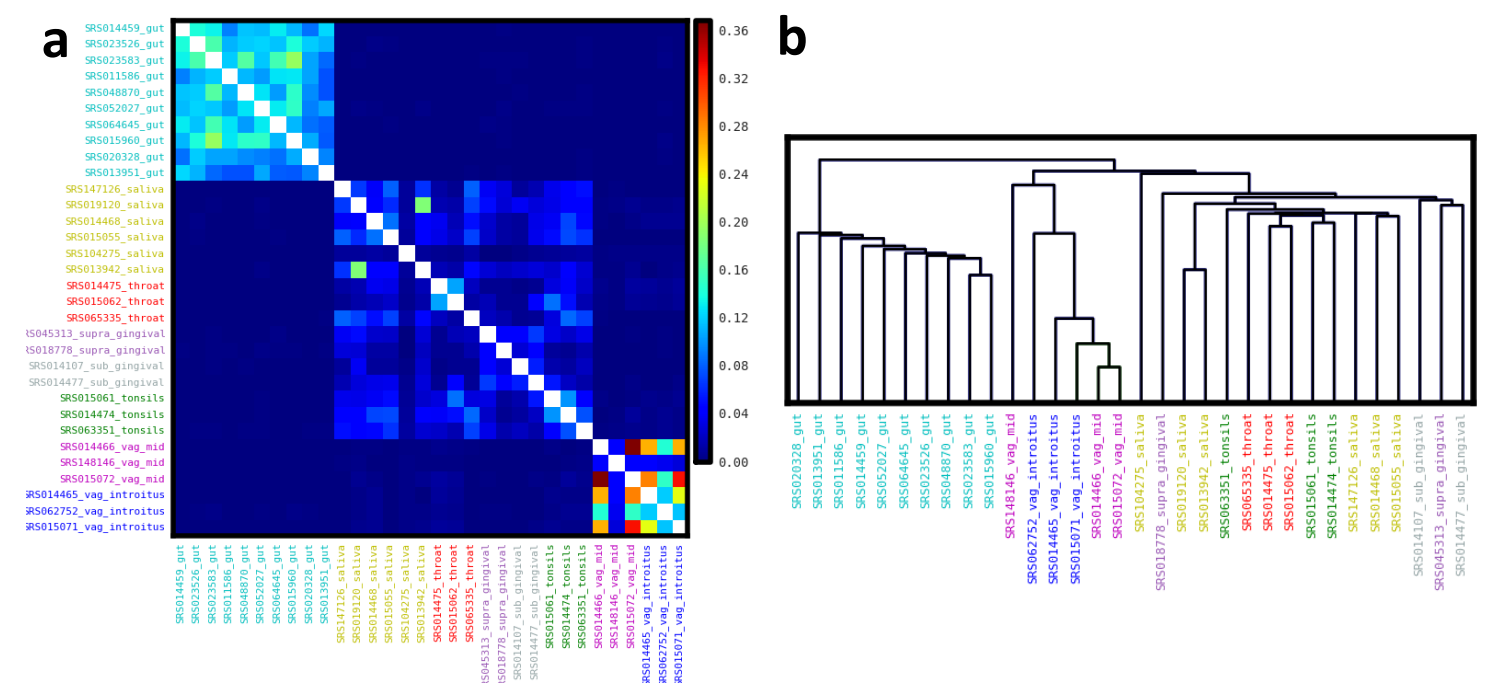

Fig. 4: Jaccard similarities estimated using MinHash for a panel of 32 HMP samples extracted from different body sites. (a) Heatmap of Jaccard similarities and (b) the resulting hierarchical clustering of the samples.

kmer counts. Furthermore, through offline indexing of publicly available cohorts of metagenomes, it enables efficient analysis of single metagenomic samples, catering to settings with scarce computational and data resources. As a result, an ungoing effort of this project is to index all the publicly-available samples from various metagenomic archives and make them available to the public.

\section{References}

1. Abbar, S., Amer-Yahia, S., Indyk, P., Mahabadi, S.: Real-time recommendation of diverse related articles. In: Proceedings of the 22nd international conference on World Wide Web. pp. 1-12. ACM (2013)

2. Albertsen, M., Hugenholtz, P., Skarshewski, A., Nielsen, K.L., Tyson, G.W., Nielsen, P.H.: Genome sequences of rare, uncultured bacteria obtained by differential coverage binning of multiple metagenomes. Nature biotechnology $31(6), 533-538(2013)$

3. Alneberg, J., Bjarnason, B.S., de Bruijn, I., Schirmer, M., Quick, J., Ijaz, U.Z., Lahti, L., Loman, N.J., Andersson, A.F., Quince, C.: Binning metagenomic contigs by coverage and composition. Nature methods 11(11), 1144-1146 (2014)

4. Bankevich, A., Nurk, S., Antipov, D., Gurevich, A.A., Dvorkin, M., Kulikov, A.S., Lesin, V.M., Nikolenko, S.I., Pham, S., Prjibelski, A.D., et al.: Spades: a new genome assembly algorithm and its applications to single-cell sequencing. Journal of Computational Biology 19(5), 455-477 (2012)

5. Boisvert, S., Laviolette, F., Corbeil, J.: Ray: simultaneous assembly of reads from a mix of high-throughput sequencing technologies. Journal of Computational Biology 17(11), 1519-1533 (2010)

6. Botelho, F.C., Pagh, R., Ziviani, N.: Simple and space-efficient minimal perfect hash functions. In: Workshop on Algorithms and Data Structures. pp. 139-150. Springer (2007)

7. Broder, A.Z., Charikar, M., Frieze, A.M., Mitzenmacher, M.: Min-wise independent permutations. Journal of Computer and System Sciences 60(3), 630-659 (2000)

8. Chen, L., Cong, G.: Diversity-aware top-k publish/subscribe for text stream. In: Proceedings of the 2015 ACM SIGMOD International Conference on Management of Data. pp. 347-362. ACM (2015)

9. Cichelli, R.J.: Minimal perfect hash functions made simple. Communications of the ACM 23(1), 17-19 (1980)

10. Erkut, E.: The discrete p-dispersion problem. European Journal of Operational Research 46(1), 48-60 (1990)

11. https://github.com/lh3/seqtk: 
12. Hunter, S., Corbett, M., Denise, H., Fraser, M., Gonzalez-Beltran, A., Hunter, C., Jones, P., Leinonen, R., McAnulla, C., Maguire, E., et al.: Ebi metagenomics - a new resource for the analysis and archiving of metagenomic data. Nucleic acids research 42(D1), D600-D606 (2014)

13. Huson, D.H., Auch, A.F., Qi, J., Schuster, S.C.: Megan analysis of metagenomic data. Genome research 17(3), 377-386 (2007)

14. Hyatt, D., Chen, G.L., LoCascio, P.F., Land, M.L., Larimer, F.W., Hauser, L.J.: Prodigal: prokaryotic gene recognition and translation initiation site identification. BMC bioinformatics 11(1), 1 (2010)

15. Indyk, P., Motwani, R.: Approximate nearest neighbors: towards removing the curse of dimensionality. In: Proceedings of the thirtieth annual ACM symposium on Theory of computing. pp. 604-613. ACM (1998)

16. Kang, D.D., Froula, J., Egan, R., Wang, Z.: Metabat, an efficient tool for accurately reconstructing single genomes from complex microbial communities. PeerJ 3, e1165 (2015)

17. Kislyuk, A., Bhatnagar, S., Dushoff, J., Weitz, J.S.: Unsupervised statistical clustering of environmental shotgun sequences. BMC bioinformatics 10(1), 1 (2009)

18. Langmead, B., Salzberg, S.L.: Fast gapped-read alignment with bowtie 2. Nature methods 9(4), 357-359 (2012)

19. Li, H., Handsaker, B., Wysoker, A., Fennell, T., Ruan, J., Homer, N., Marth, G., Abecasis, G., Durbin, R., et al.: The sequence alignment/map format and samtools. Bioinformatics 25(16), 2078-2079 (2009)

20. Lu, Y.Y., Chen, T., Fuhrman, J.A., Sun, F.: Cocacola: binning metagenomic contigs using sequence composition, read coverage, co-alignment, and paired-end read linkage. Bioinformatics p. btw290 (2016)

21. Marçais, G., Kingsford, C.: A fast, lock-free approach for efficient parallel counting of occurrences of k-mers. Bioinformatics 27(6), 764-770 (2011)

22. Murphy, K.P.: Machine learning: a probabilistic perspective. MIT press (2012)

23. Parks, D.H., Imelfort, M., Skennerton, C.T., Hugenholtz, P., Tyson, G.W.: Checkm: assessing the quality of microbial genomes recovered from isolates, single cells, and metagenomes. Genome research 25(7), 1043-1055 (2015)

24. Patro, R., Mount, S.M., Kingsford, C.: Sailfish enables alignment-free isoform quantification from rna-seq reads using lightweight algorithms. Nature biotechnology 32(5), 462-464 (2014)

25. Ravi, S., Rosenkrantz, D.J., Tayi, G.K.: Facility dispersion problems: Heuristics and special cases. In: Workshop on Algorithms and Data Structures. pp. 355-366. Springer (1991)

26. Rosen, G.L., Reichenberger, E.R., Rosenfeld, A.M.: Nbc: the naive bayes classification tool webserver for taxonomic classification of metagenomic reads. Bioinformatics 27(1), 127-129 (2011)

27. Sharon, I., Morowitz, M.J., Thomas, B.C., Costello, E.K., Relman, D.A., Banfield, J.F.: Time series community genomics analysis reveals rapid shifts in bacterial species, strains, and phage during infant gut colonization. Genome research 23(1), 111-120 (2013)

28. Strous, M., Kraft, B., Bisdorf, R., Tegetmeyer, H.: The binning of metagenomic contigs for microbial physiology of mixed cultures. Frontiers in microbiology 3, 410 (2012)

29. Turnbaugh, P.J., Ley, R.E., Hamady, M., Fraser-Liggett, C., Knight, R., Gordon, J.I.: The human microbiome project: exploring the microbial part of ourselves in a changing world. Nature 449(7164), 804 (2007)

30. Wood, D.E., Salzberg, S.L.: Kraken: ultrafast metagenomic sequence classification using exact alignments. Genome biology 15(3), 1 (2014)

31. Wu, Y.W., Tang, Y.H., Tringe, S.G., Simmons, B.A., Singer, S.W.: Maxbin: an automated binning method to recover individual genomes from metagenomes using an expectation-maximization algorithm. Microbiome 2(1), $26(2014)$

32. Zhang, Q., Pell, J., Canino-Koning, R., Howe, A.C., Brown, C.T.: These are not the k-mers you are looking for: efficient online k-mer counting using a probabilistic data structure. PloS one 9(7), e101271 (2014) 